\title{
Dilute neutron matter on the lattice at next-to-leading order in chiral effective field theory
}

\author{
Buḡra Borasoy $^{a}$, Evgeny Epelbaum ${ }^{b, a}$, Hermann Krebs ${ }^{a, b}$, Dean Lee ${ }^{c, a}$, Ulf-G. Meißner ${ }^{a, b}$ \\ ${ }^{a}$ Helmholtz-Institut für Strahlen- und Kernphysik (Theorie) Universität Bonn, \\ Nußallee 14-16, D-53115 Bonn, Germany \\ ${ }^{b}$ Institut für Kernphysik (Theorie), Forschungszentrum Jülich, D-52425 Jülich, Germany \\ ${ }^{c}$ Department of Physics, North Carolina State University, Raleigh, NC 27695, USA
}

\begin{abstract}
We discuss lattice simulations of the ground state of dilute neutron matter at nextto-leading order in chiral effective field theory. In a previous paper the coefficients of the next-to-leading-order lattice action were determined by matching nucleon-nucleon scattering data for momenta up to the pion mass. Here the same lattice action is used to simulate the ground state of up to 12 neutrons in a periodic cube using Monte Carlo. We explore the density range from $2 \%$ to $8 \%$ of normal nuclear density and analyze the ground state energy as an expansion about the unitarity limit with corrections due to finite scattering length, effective range, and $P$-wave interactions.
\end{abstract}




\section{INTRODUCTION}

This is the second of a pair of papers studying chiral effective field theory on the lattice at next-to-leading order. In the first paper [1] we used nucleon-nucleon scattering data at low energies to determine unknown operator coefficients of the next-to-leading-order lattice action. We also tested model independence of the effective theory at fixed lattice spacing by computing next-to-leading-order corrections for two different leading-order lattice actions. In this paper we use the Gaussian-smeared lattice actions $\mathrm{LO}_{2}$ and $\mathrm{NLO}_{2}$ defined in [1] to simulate dilute neutron matter in a periodic cube. We probe the density range from $2 \%$ to $8 \%$ of normal nuclear matter density. Neutron-rich matter at this density is likely present in the inner crust of neutron stars [2, 3]. The Pauli suppression of three-body forces in dilute neutron matter makes it a good testing ground for chiral effective field theory applied to many-nucleon systems.

The organization of the paper is as follows. We review the lattice interactions contained in the leading-order (LO) and next-to-leading-order (NLO) transfer matrices. These transfer matrices are rewritten in terms of one-body interactions with auxiliary fields. This allows us to simulate the ground state of the many-neutron system using transfer matrix projection and hybrid Monte Carlo. The results of the simulations are compared with published results for the ground state energy. We also analyze the ground state energy as an expansion near the unitarity limit, where the scattering length is infinite and the interactions have negligible range.

\section{LATTICE TRANSFER MATRICES WITHOUT AUXILIARY FIELDS}

In [1] we defined the lattice transfer matrix $M_{\mathrm{LO}_{2}}$ at leading order and $M_{\mathrm{NLO}_{2}}$ at next-toleading order. We use the same lattice conventions here and briefly summarize the relevant definitions in the appendix. Throughout we use spatial lattice spacing $a=(100 \mathrm{MeV})^{-1}$ and temporal lattice spacing $a_{t}=(70 \mathrm{MeV})^{-1}$. We take for our physical constants $m=$ 938.92 $\mathrm{MeV}$ as the nucleon mass, $m_{\pi}=138.08 \mathrm{MeV}$ as the pion mass, $f_{\pi}=93 \mathrm{MeV}$ as the pion decay constant, and $g_{A}=1.26$ as the nucleon axial charge. In [1] we also defined lattice actions $M_{\mathrm{LO}_{1}}$ and $M_{\mathrm{NLO}_{1}}$. Given the significant computational resources required for the Monte Carlo simulations, we use only the Gaussian-smeared actions $M_{\mathrm{LO}_{2}}$ and $M_{\mathrm{NLO}_{2}}$. 
These yield a slightly better description of the $S$-wave interactions expected to be dominant in dilute neutron matter. Since we consider only one version of the lattice action here we drop the subscript "2" and write $M_{\mathrm{LO}}$ and $M_{\mathrm{NLO}}$. Tests of model independence using different lattice actions and lattice spacings will be pursued in future studies.

The leading-order lattice transfer matrix is

$$
\begin{aligned}
M_{\mathrm{LO}} & =: \exp \left\{-H_{\text {free }} \alpha_{t}-\frac{\alpha_{t}}{2 L^{3}} \sum_{\vec{q}} f\left(q^{2}\right)\left[C \rho^{a^{\dagger}, a}(\vec{q}) \rho^{a^{\dagger}, a}(-\vec{q})+C_{I^{2}} \sum_{I} \rho_{I}^{a^{\dagger}, a}(\vec{q}) \rho_{I}^{a^{\dagger}, a}(-\vec{q})\right]\right. \\
& \left.+\frac{g_{A}^{2} \alpha_{t}^{2}}{8 f_{\pi}^{2} q_{\pi}} \sum_{S_{1}, S_{2}, I} \sum_{\vec{n}_{1}, \vec{n}_{2}} G_{S_{1} S_{2}}\left(\vec{n}_{1}-\vec{n}_{2}\right) \rho_{S_{1}, I}^{a^{\dagger}, a}\left(\vec{n}_{1}\right) \rho_{S_{2}, I}^{a^{\dagger}, a}\left(\vec{n}_{2}\right)\right\}:
\end{aligned}
$$

All of the terms appearing in Eq. (1) were defined in [1] and summarized in the appendix. The isospin of any two-neutron state is $I_{z}=-1, I=1$. Therefore only the linear combination

$$
C^{I=1}=C+C_{I^{2}}
$$

contributes to systems with only neutrons. As in [1] the coefficient $C^{I=1}$ is set to $-3.414 \times$ $10^{-5} \mathrm{MeV}^{-2}$.

At next-to-leading order the lattice transfer matrix is

$$
\begin{aligned}
M_{\mathrm{NLO}} & =: \exp \left\{-H_{\mathrm{free}} \alpha_{t}-\frac{\alpha_{t}}{2 L^{3}} \sum_{\vec{q}} f\left(q^{2}\right)\left[C \rho^{a^{\dagger}, a}(\vec{q}) \rho^{a^{\dagger}, a}(-\vec{q})+C_{I^{2}} \sum_{I} \rho_{I}^{a^{\dagger}, a}(\vec{q}) \rho_{I}^{a^{\dagger}, a}(-\vec{q})\right]\right. \\
& -\alpha_{t}\left[\Delta V+\Delta V_{I^{2}}+V_{q^{2}}+V_{I^{2}, q^{2}}+V_{S^{2}, q^{2}}+V_{S^{2}, I^{2}, q^{2}}+V_{(q \cdot S)^{2}}+V_{I^{2},(q \cdot S)^{2}}+V_{(i q \times S) \cdot k}^{I=1}\right] \\
& \left.+\frac{g_{A}^{2} \alpha_{t}^{2}}{8 f_{\pi}^{2} q_{\pi}} \sum_{S_{1}, S_{2}, I} \sum_{\vec{n}_{1}, \vec{n}_{2}} G_{S_{1} S_{2}}\left(\vec{n}_{1}-\vec{n}_{2}\right) \rho_{S_{1}, I}^{a^{\dagger}, a}\left(\vec{n}_{1}\right) \rho_{S_{2}, I}^{a^{\dagger}, a}\left(\vec{n}_{2}\right)\right\}
\end{aligned}
$$

The NLO corrections to the leading-order contact interactions are

$$
\begin{gathered}
\Delta V=\frac{1}{2} \Delta C: \sum_{\vec{n}} \rho^{a^{\dagger}, a}(\vec{n}) \rho^{a^{\dagger}, a}(\vec{n}):, \\
\Delta V_{I^{2}}=\frac{1}{2} \Delta C_{I^{2}}: \sum_{\vec{n}, I} \rho_{I}^{a^{\dagger}, a}(\vec{n}) \rho_{I}^{a^{\dagger}, a}(\vec{n}): .
\end{gathered}
$$

Again only the $I=1$ combination contributes to neutron-neutron scattering,

$$
\Delta C^{I=1}=\Delta C+\Delta C_{I^{2}} .
$$


There are seven independent NLO contact interactions with two derivatives,

$$
\begin{aligned}
& V_{q^{2}}=-\frac{1}{2} C_{q^{2}}: \sum_{\vec{n}, l} \rho^{a^{\dagger}, a}(\vec{n}) \nabla_{l}^{2} \rho^{a^{\dagger}, a}(\vec{n}): \\
& V_{I^{2}, q^{2}}=-\frac{1}{2} C_{I^{2}, q^{2}}: \sum_{\vec{n}, I, l} \rho_{I}^{a^{\dagger}, a}(\vec{n}) \nabla_{l}^{2} \rho_{I}^{a^{\dagger}, a}(\vec{n}): \\
& V_{S^{2}, q^{2}}=-\frac{1}{2} C_{S^{2}, q^{2}}: \sum_{\vec{n}, S, l} \rho_{S}^{a^{\dagger}, a}(\vec{n}) \nabla_{l}^{2} \rho_{S}^{a^{\dagger}, a}(\vec{n}): \\
& V_{S^{2}, I^{2}, q^{2}}=-\frac{1}{2} C_{S^{2}, I^{2}, q^{2}}: \sum_{\vec{n}, S, I, l} \rho_{S, I}^{a^{\dagger}, a}(\vec{n}) \nabla_{l}^{2} \rho_{S, I}^{a^{\dagger}, a}(\vec{n}): \\
& V_{(q \cdot S)^{2}}=\frac{1}{2} C_{(q \cdot S)^{2}}: \sum_{\vec{n}} \sum_{S} \Delta_{S} \rho_{S}^{a^{\dagger}, a}(\vec{n}) \sum_{S^{\prime}} \Delta_{S^{\prime}} \rho_{S^{\prime}}^{a^{\dagger}, a}(\vec{n}): \\
& V_{I^{2},(q \cdot S)^{2}}=\frac{1}{2} C_{I^{2},(q \cdot S)^{2}}: \sum_{\vec{n}, I} \sum_{S} \Delta_{S} \rho_{S, I}^{a^{\dagger}, a}(\vec{n}) \sum_{S^{\prime}} \Delta_{S^{\prime}} \rho_{S^{\prime}, I}^{a^{\dagger}, a}(\vec{n}):, \\
& V_{(i q \times S) \cdot k}^{I=1}=-\frac{i}{2} C_{(i q \times S) \cdot k}^{I=1}\left\{\frac{3}{4}: \sum_{\vec{n}, l, S, l^{\prime}} \varepsilon_{l, S, l^{\prime}}\left[\Pi_{l}^{a^{\dagger}, a}(\vec{n}) \Delta_{l^{\prime}} \rho_{S}^{a^{\dagger}, a}(\vec{n})+\Pi_{l, S}^{a^{\dagger}, a}(\vec{n}) \Delta_{l^{\prime}} \rho^{a^{\dagger}, a}(\vec{n})\right]:\right. \\
& \left.+\frac{1}{4}: \sum_{\vec{n}, l, S, l^{\prime}, I} \varepsilon_{l, S, l^{\prime}}\left[\Pi_{l, I}^{a^{\dagger}, a}(\vec{n}) \Delta_{l^{\prime}} \rho_{S, I}^{a^{\dagger}, a}(\vec{n})+\Pi_{l, S, I}^{a^{\dagger}, a}(\vec{n}) \Delta_{l^{\prime}} \rho_{I}^{a^{\dagger}, a}(\vec{n})\right]:\right\} .
\end{aligned}
$$

The various static densities, current densities, and symbols $\Delta_{l}$ and $\nabla_{l}^{2}$, are defined in the appendix. The $V_{(i q \times S) \cdot k}^{I=1}$ interaction is already projected onto $I=1$. The other interactions give three independent $I=1$ coefficients,

$$
\begin{gathered}
C_{q^{2}}^{I=1}=C_{q^{2}}+C_{I^{2}, q^{2}}, \\
C_{S^{2}, q^{2}}^{I=1}=C_{S^{2}, q^{2}}+C_{S^{2}, I^{2}, q^{2}}, \\
C_{(q \cdot S)^{2}}^{I=1}=C_{(q \cdot S)^{2}}+C_{I^{2},(q \cdot S)^{2}} .
\end{gathered}
$$

There are a total of five independent $I=1$ coefficients at NLO. These were computed in [1] using the spherical wall method [4]. The values of the $I=1$ coefficients are shown in Table I. While the LO terms in the transfer matrix are iterated nonperturbatively, the contribution from each NLO interaction is computed using first-order perturbation theory. In Fig. 1 the resulting scattering phase shifts for the $I=1$ singlet $S$-wave and triplet $P$-waves are shown together with partial wave results from [5]. The five arrows show data points used 
TABLE I: Results for $I=1$ NLO operator coefficients

\begin{tabular}{||l|l||}
\hline \hline$\Delta C^{I=1}$ & $-7.7 \times 10^{-7} \mathrm{MeV}^{-2}$ \\
\hline$C_{q^{2}}^{I=1}$ & $-1.42 \times 10^{-9} \mathrm{MeV}^{-4}$ \\
\hline$C_{S^{2}, q^{2}}^{I=1}$ & $-4.53 \times 10^{-10} \mathrm{MeV}^{-4}$ \\
\hline$C_{(q \cdot S)^{2}}^{I=1}$ & $-1.80 \times 10^{-10} \mathrm{MeV}^{-4}$ \\
\hline$C_{(i q \times S) \cdot k}^{I=1}$ & $9.81 \times 10^{-11} \mathrm{MeV}^{-4}$ \\
\hline
\end{tabular}

to determine the five $I=1$ NLO coefficients. There are also four other data points used in [1] to determine the four $I=0$ coefficients, but these are irrelevant for neutron-neutron scattering.

Up until this point our lattice formalism has been constructed for a general system of low-energy nucleons. For reasons of numerical efficiency for the Monte Carlo simulation we now specialize to the case where all nucleons are neutrons. With this restriction the following replacements are possible:

$$
\begin{gathered}
C \rho^{a^{\dagger}, a}(\vec{q}) \rho^{a^{\dagger}, a}(-\vec{q})+C_{I^{2}} \sum_{I} \rho_{I}^{a^{\dagger}, a}(\vec{q}) \rho_{I}^{a^{\dagger}, a}(-\vec{q}) \rightarrow C^{I=1} \rho^{a^{\dagger}, a}(\vec{q}) \rho^{a^{\dagger}, a}(-\vec{q}), \\
\sum_{S_{1}, S_{2}, I} \sum_{\vec{n}_{1}, \vec{n}_{2}} G_{S_{1} S_{2}}\left(\vec{n}_{1}-\vec{n}_{2}\right) \rho_{S_{1}, I}^{a^{\dagger}, a}\left(\vec{n}_{1}\right) \rho_{S_{2}, I}^{a^{\dagger}, a}\left(\vec{n}_{2}\right) \rightarrow \sum_{S_{1}, S_{2}} \sum_{\vec{n}_{1}, \vec{n}_{2}} G_{S_{1} S_{2}}\left(\vec{n}_{1}-\vec{n}_{2}\right) \rho_{S_{1}}^{a^{\dagger}, a}\left(\vec{n}_{1}\right) \rho_{S_{2}}^{a^{\dagger}, a}\left(\vec{n}_{2}\right) .
\end{gathered}
$$

This change has no effect on the interactions between neutrons but leads to the simplified transfer matrix,

$$
\begin{aligned}
M_{\mathrm{LO}} & \rightarrow: \exp \left\{-H_{\text {free }} \alpha_{t}-\frac{C^{I=1} \alpha_{t}}{2 L^{3}} \sum_{\vec{q}} f\left(q^{2}\right) \rho^{a^{\dagger}, a}(\vec{q}) \rho^{a^{\dagger}, a}(-\vec{q})\right. \\
& \left.+\frac{g_{A}^{2} \alpha_{t}^{2}}{8 f_{\pi}^{2} q_{\pi}} \sum_{S_{1}, S_{2}} \sum_{\vec{n}_{1}, \vec{n}_{2}} G_{S_{1} S_{2}}\left(\vec{n}_{1}-\vec{n}_{2}\right) \rho_{S_{1}}^{a^{\dagger}, a}\left(\vec{n}_{1}\right) \rho_{S_{2}}^{a^{\dagger}, a}\left(\vec{n}_{2}\right)\right\}:
\end{aligned}
$$



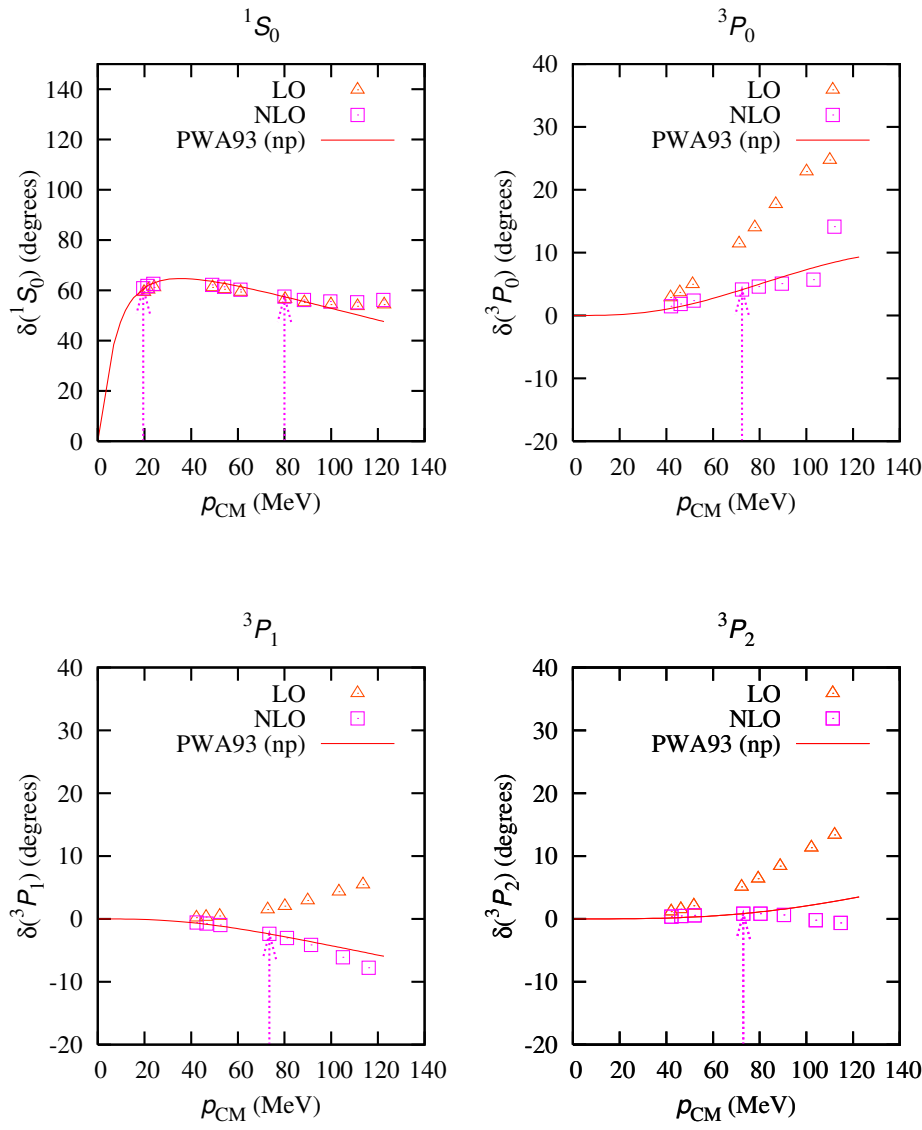

FIG. 1: Scattering phase shifts for the $I=1$ singlet $S$-wave and triplet $P$-waves versus centerof-mass momentum. The five arrows show data points used to determine the five $I=1$ NLO coefficients.

At next-to-leading order the simplified neutron transfer matrix is

$$
\begin{aligned}
M_{\mathrm{NLO}} & \rightarrow: \exp \left\{-H_{\mathrm{free}} \alpha_{t}-\frac{C^{I=1} \alpha_{t}}{2 L^{3}} \sum_{\vec{q}} f\left(q^{2}\right) \rho^{a^{\dagger}, a}(\vec{q}) \rho^{a^{\dagger}, a}(-\vec{q})\right. \\
& -\alpha_{t}\left[\frac{\Delta C^{I=1}}{\Delta C} \Delta V+\frac{C_{q^{2}}^{I=1}}{C_{q^{2}}} V_{q^{2}}+\frac{C_{S^{2}, q^{2}}^{I=1}}{C_{S^{2}, q^{2}}} V_{S^{2}, q^{2}}+\frac{C_{(q \cdot S)^{2}}^{I=1}}{C_{(q \cdot S)^{2}}} V_{(q \cdot S)^{2}}+V_{(i q \times S) \cdot k}^{I=1}\right] \\
& \left.+\frac{g_{A}^{2} \alpha_{t}^{2}}{8 f_{\pi}^{2} q_{\pi}} \sum_{S_{1}, S_{2}, \vec{n}_{1}, \vec{n}_{2}} G_{S_{1} S_{2}}\left(\vec{n}_{1}-\vec{n}_{2}\right) \rho_{S_{1}}^{a^{\dagger}, a}\left(\vec{n}_{1}\right) \rho_{S_{2}}^{a^{\dagger}, a}\left(\vec{n}_{2}\right)\right\}:
\end{aligned}
$$

In the following we use these simplified forms for the leading-order and next-to-leading-order transfer matrices. 


\section{LATTICE TRANSFER MATRICES WITH AUXILIARY FIELDS}

The transfer matrices in Eq. (19) and (20) can be rewritten as one-body interactions with auxiliary fields. This auxiliary-field formulation is useful for the many-body simulation. The exact equivalence between lattice formalisms with and without auxiliary fields was shown in [6, 7]. We summarize the results here.

In neutron-neutron scattering only the neutral pion contributes to one-pion exchange. Up to this point we have been writing the rescaled neutral pion field as $\pi_{3}^{\prime}$. In the following we drop the subscript " 3 " and simply write $\pi^{\prime}$. Let $M^{\left(n_{t}\right)}\left(\pi^{\prime}, s\right)$ be the leading-order auxiliaryfield transfer matrix at time step $n_{t}$,

$$
\begin{aligned}
M^{\left(n_{t}\right)}\left(\pi^{\prime}, s\right)=: \exp \{ & -H_{\text {free }} \alpha_{t}+\frac{g_{A} \alpha_{t}}{2 f_{\pi} \sqrt{q_{\pi}}} \sum_{S} \Delta_{S} \pi^{\prime}\left(\vec{n}, n_{t}\right) \rho_{S}^{a^{\dagger}, a}(\vec{n}) \\
& \left.+\sqrt{-C^{I=1} \alpha_{t}} \sum_{\vec{n}} s\left(\vec{n}, n_{t}\right) \rho^{a^{\dagger}, a}(\vec{n})\right\}:
\end{aligned}
$$

We can write $M_{\mathrm{LO}}$ as the normalized integral

$$
M_{\mathrm{LO}}=\frac{\int D \pi^{\prime} D s e^{-S_{\pi \pi}^{\left(n_{t}\right)}-S_{s s}^{\left(n_{t}\right)}} M^{\left(n_{t}\right)}\left(\pi^{\prime}, s\right)}{\int D \pi^{\prime} D s e^{-S_{\pi \pi}^{\left(n_{t}\right)}-S_{s s}^{\left(n_{t}\right)}}},
$$

where $S_{\pi \pi}^{\left(n_{t}\right)}$ is the piece of the instantaneous pion action in Eq. (A18) containing the neutral pion field at time step $n_{t}$,

$$
S_{\pi \pi}^{\left(n_{t}\right)}\left(\pi^{\prime}\right)=\frac{1}{2} \sum_{\vec{n}} \pi^{\prime}\left(\vec{n}, n_{t}\right) \pi^{\prime}\left(\vec{n}, n_{t}\right)-\frac{\alpha_{t}}{q_{\pi}} \sum_{\vec{n}, l} \pi^{\prime}\left(\vec{n}, n_{t}\right) \pi^{\prime}\left(\vec{n}+\hat{l}, n_{t}\right),
$$

and $S_{s s}^{\left(n_{t}\right)}$ is the auxiliary-field action at time step $n_{t}$,

$$
S_{s s}^{\left(n_{t}\right)}=\frac{1}{2} \sum_{\vec{n}, \vec{n}^{\prime}} s\left(\vec{n}, n_{t}\right) f^{-1}\left(\vec{n}-\vec{n}^{\prime}\right) s\left(\vec{n}^{\prime}, n_{t}\right)
$$

with

$$
f^{-1}\left(\vec{n}-\vec{n}^{\prime}\right)=\frac{1}{L^{3}} \sum_{\vec{q}} \frac{1}{f\left(q^{2}\right)} e^{-i \vec{q} \cdot\left(\vec{n}-\vec{n}^{\prime}\right)}
$$


The NLO interactions require several additional auxiliary fields. Let

$$
\begin{aligned}
U^{\left(n_{t}\right)}(\varepsilon) & =\sum_{\vec{n}} \varepsilon_{\rho}\left(\vec{n}, n_{t}\right) \rho^{a^{\dagger}, a}(\vec{n})+\sum_{\vec{n}, S} \varepsilon_{\rho_{S}}\left(\vec{n}, n_{t}\right) \rho_{S}^{a^{\dagger}, a}(\vec{n})+\sum_{\vec{n}, S} \varepsilon_{\Delta_{S} \rho}\left(\vec{n}, n_{t}\right) \Delta_{S} \rho^{a^{\dagger}, a}(\vec{n}) \\
& +\sum_{\vec{n}, S, S^{\prime}} \varepsilon_{\Delta_{S} \rho_{S^{\prime}}}\left(\vec{n}, n_{t}\right) \Delta_{S} \rho_{S^{\prime}}^{a^{\dagger}, a}(\vec{n})+\sum_{\vec{n}, l} \varepsilon_{\nabla_{l}^{2} \rho}\left(\vec{n}, n_{t}\right) \nabla_{l}^{2} \rho^{a^{\dagger}, a}(\vec{n}) \\
& +\sum_{\vec{n}, l, S} \varepsilon_{\nabla_{l}^{2} \rho_{S}}\left(\vec{n}, n_{t}\right) \nabla_{l}^{2} \rho_{S}^{a^{\dagger}, a}(\vec{n})+\sum_{\vec{n}, l} \varepsilon_{\Pi_{l}}\left(\vec{n}, n_{t}\right) \Pi_{l}^{a^{\dagger}, a}(\vec{n})+\sum_{\vec{n}, l, S} \varepsilon_{\Pi_{l, S}}\left(\vec{n}, n_{t}\right) \Pi_{l, S}^{a^{\dagger}, a}(\vec{n}) .
\end{aligned}
$$

With these extra fields and linear functional $U^{\left(n_{t}\right)}(\varepsilon)$ we define

$$
\begin{aligned}
M^{\left(n_{t}\right)}\left(\pi^{\prime}, s, \varepsilon\right)=: \exp \{ & -H_{\text {free }} \alpha_{t}+\frac{g_{A} \alpha_{t}}{2 f_{\pi} \sqrt{q_{\pi}}} \sum_{S} \Delta_{S} \pi^{\prime}\left(\vec{n}, n_{t}\right) \rho_{S}^{a^{\dagger}, a}(\vec{n}) \\
& \left.+\sqrt{-C^{I=1} \alpha_{t}} \sum_{\vec{n}} s\left(\vec{n}, n_{t}\right) \rho^{a^{\dagger}, a}(\vec{n})+\sqrt{\alpha_{t}} U^{\left(n_{t}\right)}(\varepsilon)\right\}: .
\end{aligned}
$$

We also define the normalized integral,

$$
M^{\left(n_{t}\right)}(\varepsilon)=\frac{\int D \pi^{\prime} D s e^{-S_{\pi \pi}^{\left(n_{t}\right)}-S_{s s}^{\left(n_{t}\right)}} M^{\left(n_{t}\right)}\left(\pi^{\prime}, s, \varepsilon\right)}{\int D \pi^{\prime} D s e^{-S_{\pi \pi}^{\left(n_{t}\right)}-S_{s s}^{\left(n_{t}\right)}}} .
$$

When all $\varepsilon$ fields are set to zero we recover $M_{\mathrm{LO}}$,

$$
M^{\left(n_{t}\right)}(0)=M_{\mathrm{LO}}
$$

To first order the NLO interactions in $M_{\mathrm{NLO}}$ can be written as a sum of bilinear derivatives of $M^{\left(n_{t}\right)}(\varepsilon)$ with respect to the $\varepsilon$ fields at $\varepsilon=0$,

$$
\begin{aligned}
M_{\mathrm{NLO}} & =M_{\mathrm{LO}} \\
& -\left.\frac{1}{2} \Delta C^{I=1} \sum_{\vec{n}} \frac{\delta}{\delta \varepsilon_{\rho}\left(\vec{n}, n_{t}\right)} \frac{\delta}{\delta \varepsilon_{\rho}\left(\vec{n}, n_{t}\right)} M^{\left(n_{t}\right)}(\varepsilon)\right|_{\varepsilon=0} \\
& +\left.\frac{1}{2} C_{q^{2}}^{I=1} \sum_{\vec{n}} \frac{\delta}{\delta \varepsilon_{\rho}\left(\vec{n}, n_{t}\right)} \frac{\delta}{\delta \varepsilon_{\nabla_{l}^{2} \rho}\left(\vec{n}, n_{t}\right)} M^{\left(n_{t}\right)}(\varepsilon)\right|_{\varepsilon=0}+\cdots
\end{aligned}
$$

\section{TRANSFER MATRIX PROJECTION METHOD}

We use the transfer matrix projection method introduced in [8]. First we give a short overview using simple continuum notation. Let $\left|\Psi^{\text {free }}\right\rangle$ be a Slater determinant of freeparticle standing waves in a periodic cube for $N$ neutrons. Let $H_{\mathrm{LO}}$ be the Hamiltonian at 
leading order, and $H_{\mathrm{NLO}}$ be the Hamiltonian at next-to-leading order. Let $H_{\mathrm{SU}(2) \not t}$ be the same as $H_{\mathrm{LO}}$, but with one-pion exchange turned off by setting $g_{A}$ to zero. As the notation suggests, $H_{\mathrm{SU}(2) \not t}$ is invariant under an exact $\mathrm{SU}(2)$ intrinsic-spin symmetry.

Let us define a trial wavefunction

$$
\left|\Psi\left(t^{\prime}\right)\right\rangle=\exp \left(-H_{\mathrm{SU}(2) \not t^{\prime}}\right)\left|\Psi^{\text {free }}\right\rangle .
$$

In this approach $\exp \left(-H_{\mathrm{SU}(2) \pi t^{\prime}}\right)$ acts as an approximate low-energy filter. In the auxiliaryfield Monte Carlo calculation this part of the Euclidean time propagation is positive definite for any even number of neutrons invariant under the $\mathrm{SU}(2)$ intrinsic-spin symmetry [9, 10, 11]. With this trial wavefunction we define the amplitude,

$$
Z(t)=\left\langle\Psi\left(t^{\prime}\right)\left|\exp \left(-H_{\mathrm{LO}} t\right)\right| \Psi\left(t^{\prime}\right)\right\rangle,
$$

as well as the transient energy,

$$
E_{\mathrm{LO}}(t)=-\frac{\partial}{\partial t}[\ln Z(t)]
$$

In limit of large Euclidean time $t$ we get

$$
\lim _{t \rightarrow \infty} E_{\mathrm{LO}}(t)=E_{0, \mathrm{LO}}
$$

where $E_{0, \mathrm{LO}}$ is the energy of the lowest eigenstate $\left|\Psi_{0}\right\rangle$ of $H_{\mathrm{LO}}$ with nonzero inner product with $\left|\Psi\left(t^{\prime}\right)\right\rangle$.

To compute the expectation value of some operator $O$ we define

$$
Z_{O}(t)=\left\langle\Psi\left(t^{\prime}\right)\left|\exp \left(-H_{\mathrm{LO}} t / 2\right) O \exp \left(-H_{\mathrm{LO}} t / 2\right)\right| \Psi\left(t^{\prime}\right)\right\rangle .
$$

The expectation value of $O$ for $\left|\Psi_{0}\right\rangle$ is given by the large $t$ limit,

$$
\lim _{t \rightarrow \infty} \frac{Z_{O}(t)}{Z(t)}=\left\langle\Psi_{0}|O| \Psi_{0}\right\rangle
$$

Let $H_{\mathrm{NLO}}$ be the next-to-leading-order Hamiltonian. Corrections to the energy at next-toleading order can be computed using $O=H_{\mathrm{NLO}}-H_{\mathrm{LO}}$. Then

$$
\lim _{t \rightarrow \infty} \frac{Z_{O}(t)}{Z(t)}=E_{0, \mathrm{NLO}}-E_{0, \mathrm{LO}},
$$

where $E_{0, \mathrm{NLO}}$ is the ground state energy at next-to-leading order. 
On the lattice we construct $\left|\Psi\left(t^{\prime}\right)\right\rangle$ using

$$
\left|\Psi\left(t^{\prime}\right)\right\rangle=\left(M_{\mathrm{SU}(2) \not x^{\prime}}\right)^{L_{t_{o}}}\left|\Psi^{\text {free }}\right\rangle
$$

where $t^{\prime}=L_{t_{o}} \alpha_{t}$ and $L_{t_{o}}$ is the number of "outer" time steps. The amplitude $Z(t)$ is constructed using

$$
Z(t)=\left\langle\Psi\left(t^{\prime}\right)\left|\left(M_{\mathrm{LO}}\right)^{L_{t_{i}}}\right| \Psi\left(t^{\prime}\right)\right\rangle
$$

where $t=L_{t_{i}} \alpha_{t}$ and $L_{t_{i}}$ is the number of "inner" time steps. The transient energy

$$
E_{\mathrm{LO}}\left(t+\alpha_{t} / 2\right)
$$

is given by the ratio of the amplitudes for $t$ and $t+\alpha_{t}$,

$$
e^{-E_{\mathrm{LO}}\left(t+\alpha_{t} / 2\right) \cdot \alpha_{t}}=\frac{Z\left(t+\alpha_{t}\right)}{Z(t)}
$$

The ground state energy $E_{0, \mathrm{LO}}$ equals the asymptotic limit,

$$
E_{0, \mathrm{LO}}=\lim _{t \rightarrow \infty} E_{\mathrm{LO}}\left(t+\alpha_{t} / 2\right)
$$

For the ground state energy at NLO we compute expectation values of $M_{\mathrm{NLO}}$ and $M_{\mathrm{LO}}$ inserted in the middle of a string of LO transfer matrices,

$$
\begin{gathered}
Z_{M_{\mathrm{NLO}}}(t)=\left\langle\Psi\left(t^{\prime}\right)\left|\left(M_{\mathrm{LO}}\right)^{L_{t_{i}} / 2} M_{\mathrm{NLO}}\left(M_{\mathrm{LO}}\right)^{L_{t_{i}} / 2}\right| \Psi\left(t^{\prime}\right)\right\rangle, \\
Z_{M_{\mathrm{LO}}}(t)=\left\langle\Psi\left(t^{\prime}\right)\left|\left(M_{\mathrm{LO}}\right)^{L_{t_{i}} / 2} M_{\mathrm{LO}}\left(M_{\mathrm{LO}}\right)^{L_{t_{i}} / 2}\right| \Psi\left(t^{\prime}\right)\right\rangle .
\end{gathered}
$$

Clearly $Z_{M_{\mathrm{LO}}}(t)$ is the same as $Z\left(t+\alpha_{t}\right)$. We use the ratio of amplitudes,

$$
\frac{Z_{M_{\mathrm{NLO}}}(t)}{Z_{M_{\mathrm{LO}}}(t)}=1-\Delta E_{\mathrm{NLO}}(t) \alpha_{t}+\cdots,
$$

to define the transient NLO energy correction $\Delta E_{\mathrm{NLO}}(t)$. The ellipsis denotes terms which are beyond first order in the NLO coefficients. The NLO ground state energy $E_{0, \mathrm{NLO}}$ is calculated using

$$
E_{0, \mathrm{NLO}}=E_{0, \mathrm{LO}}+\lim _{t \rightarrow \infty} \Delta E_{0, \mathrm{NLO}}(t),
$$

The Monte Carlo simulation is carried out using the auxiliary-field formulations of the transfer matrices. Integrations over auxiliary and pion field configurations are computed using hybrid Monte Carlo with endpoint importance sampling. Details of this method can be found in the literature [6, 7, 8]. 
TABLE II: Monte Carlo results versus exact transfer matrix calculations for the two-neutron spin singlet $S=0$ and spin triplet $S=1$.

\begin{tabular}{|c|c|c|c|c|}
\hline & $S=0(\mathrm{MC})$ & $S=0$ (exact) & $S=1(\mathrm{MC})$ & $S=1$ (exact) \\
\hline$E_{\mathrm{LO}}\left(t+\alpha_{t} / 2\right)[\mathrm{MeV}]$ & $-2.93(2)$ & -2.9242 & $24.99(10)$ & 25.030 \\
\hline$\frac{\partial\left(\Delta E_{\mathrm{NLO}}(t)\right)}{\partial\left(\Delta C^{I=1}\right)}\left[10^{4} \mathrm{MeV}^{3}\right]$ & $4.869(6)$ & 4.8620 & $0.0003(2)$ & 0 \\
\hline$\frac{\partial\left(\Delta E_{\mathrm{NLO}}(t)\right)}{\partial\left(C_{q^{2}}^{I I}\right)}\left[10^{9} \mathrm{MeV}^{5}\right]$ & $1.617(3)$ & 1.6140 & $-1.853(4)$ & -1.8524 \\
\hline$\frac{\partial\left(\Delta E_{\mathrm{NLO}}(t)\right)}{\partial\left(C_{S^{2}, q^{2}}^{I=1}\right)}\left[10^{9} \mathrm{MeV}^{5}\right]$ & $-4.85(1)$ & -4.8419 & $-1.851(4)$ & -1.8524 \\
\hline$\frac{\partial\left(\Delta E_{\mathrm{NLO}}(t)\right)}{\partial\left(C_{(q \cdot S)^{2}}^{I=1}\right)}\left[10^{8} \mathrm{MeV}^{5}\right]$ & $-6.00(1)$ & -5.9822 & $7.00(2)$ & 7.0012 \\
\hline$\frac{\partial\left(\Delta E_{\mathrm{NLO}}(t)\right)}{\partial\left(C_{(i q \times S) \cdot k}^{I=1}\right)}\left[10^{7} \mathrm{MeV}^{5}\right]$ & $0.011(7)$ & 0 & $7.8(1)$ & 7.8743 \\
\hline$\Delta E_{\mathrm{NLO}}(t)[\mathrm{MeV}]$ & $-0.0252(3)$ & -0.025025 & $3.349(7)$ & 3.3490 \\
\hline
\end{tabular}

\section{PRECISION TESTS}

We use systems of two neutrons to test the auxiliary-field Monte Carlo simulations. We calculate the same observables using both the Monte Carlo code and the exact transfer matrix without auxiliary fields. We choose a small system so that stochastic errors are small enough to expose disagreement at the $0.1 \%-1 \%$ level. We choose the spatial length of lattice to be $L=4$ and set the outer time steps $L_{t_{o}}=2$ and inner time steps $L_{t_{i}}=4$.

For the first test we choose $\left|\Psi^{\text {free }}\right\rangle$ to be a spin-singlet state built from the Slater determinant of standing waves $\left|\psi_{1}\right\rangle$ and $\left|\psi_{2}\right\rangle$ with

$$
\left\langle 0\left|a_{i, j}(\vec{n})\right| \psi_{1}\right\rangle \propto \delta_{i, 0} \delta_{j, 1}, \quad\left\langle 0\left|a_{i, j}(\vec{n})\right| \psi_{2}\right\rangle \propto \delta_{i, 1} \delta_{j, 1}
$$

For the second test we choose a spin-triplet state with standing waves

$$
\left\langle 0\left|a_{i, j}(\vec{n})\right| \psi_{1}\right\rangle \propto \cos \left(\frac{2 \pi n_{1}}{L}\right) \delta_{i, 0} \delta_{j, 1}, \quad\left\langle 0\left|a_{i, j}(\vec{n})\right| \psi_{2}\right\rangle \propto \sin \left(\frac{2 \pi n_{1}}{L}\right) \delta_{i, 0} \delta_{j, 1}
$$

Comparisons between Monte Carlo results (MC) and exact transfer matrix calculations (exact) are shown in Table II. The numbers in parentheses are the estimated stochastic errors. We see that in each case the agreement is comparable to the estimated stochastic error. 


\section{RESULTS}

We simulate $N=8$ and $N=12$ neutrons on periodic cube lattices with spatial length $L=5,6,7$ lattice units. For each value of $N$ and $L$ we set $L_{t_{o}}=10$ and vary $L_{t_{i}}$ from 2 to 12. For $\left|\Psi^{\text {free }}\right\rangle$ we take the Slater determinant formed by standing waves

$$
\left\langle 0\left|a_{i, j}(\vec{n})\right| \psi_{2 k+1}\right\rangle \propto f_{k}(\vec{n}) \delta_{i, 0} \delta_{j, 1}, \quad\left\langle 0\left|a_{i, j}(\vec{n})\right| \psi_{2 k+2}\right\rangle \propto f_{k}(\vec{n}) \delta_{i, 1} \delta_{j, 1},
$$

where

$$
\begin{gathered}
f_{0}(\vec{n})=1, \quad f_{1}(\vec{n})=\cos \left(\frac{2 \pi n_{1}}{L}\right), \quad f_{2}(\vec{n})=\sin \left(\frac{2 \pi n_{1}}{L}\right), \\
f_{3}(\vec{n})=\cos \left(\frac{2 \pi n_{2}}{L}\right), \quad f_{4}(\vec{n})=\sin \left(\frac{2 \pi n_{2}}{L}\right), \quad f_{5}(\vec{n})=\cos \left(\frac{2 \pi n_{3}}{L}\right) .
\end{gathered}
$$

For $N=8$ we use $k=0,1, \cdots, 3$, and for $N=12$ we take $k=0,1, \cdots, 5$. For each value of $L_{t_{i}}$ a total of about $10^{6}$ hybrid Monte Carlo trajectories are generated by 1024 processors, each running completely independent trajectories. Averages and stochastic errors are computed by comparing the results of all 1024 processors.

Let $E_{0}^{\text {free }}$ be the energy of the ground state for noninteracting neutrons. In Fig. 2 we show the dimensionless ratios

$$
\frac{E_{\mathrm{LO}}(t)}{E_{0}^{\text {free }}}, \frac{\Delta E_{\mathrm{NLO}}(t)}{E_{0}^{\text {free }}}, \frac{E_{\mathrm{LO}}(t)+\Delta E_{\mathrm{NLO}}(t)}{E_{0}^{\text {free }}},
$$

versus Euclidean time $t$ for $N=8$ and $L=5,6,7$. These are labelled using the shorthand LO, $\triangle$ NLO, and NLO respectively. The same quantities are shown in Fig. 3 for $N=12$. The lattice calculations for $\Delta E_{\mathrm{NLO}}(t)$ require an even number of time steps and so fewer data points are available. In addition to the Monte Carlo data we plot the asymptotic expressions,

$$
\begin{gathered}
\frac{E_{\mathrm{LO}}(t)}{E_{0}^{\text {free }}} \approx \frac{E_{0, \mathrm{LO}}}{E_{0}^{\text {free }}}+A e^{-\delta E \cdot t}, \\
\frac{\Delta E_{\mathrm{NLO}}(t)}{E_{0}^{\text {free }}} \approx \frac{E_{0, \mathrm{NLO}}-E_{0, \mathrm{LO}}}{E_{0}^{\text {free }}}+B e^{-\delta E \cdot t / 2} . \\
\frac{E_{\mathrm{LO}}(t)+\Delta E_{\mathrm{NLO}}(t)}{E_{0}^{\text {free }}} \approx \frac{E_{0, \mathrm{NLO}}}{E_{0}^{\text {free }}}+A e^{-\delta E \cdot t}+B e^{-\delta E \cdot t / 2} .
\end{gathered}
$$

The unknown coefficients $A$ and $B$, energy gap $\delta E$, and ground state energies $E_{0, \mathrm{LO}}$ and $E_{0, \mathrm{NLO}}$ are determined by least squares fitting. The $e^{-\delta E \cdot t}$ dependence in Eq. (52) comes from the contribution of the lowest excited state with energy $\delta E$ above the ground state. The $e^{-\delta E \cdot t / 2}$ dependence in Eq. (53) comes from the matrix element of $M_{\mathrm{NLO}}$ between the 

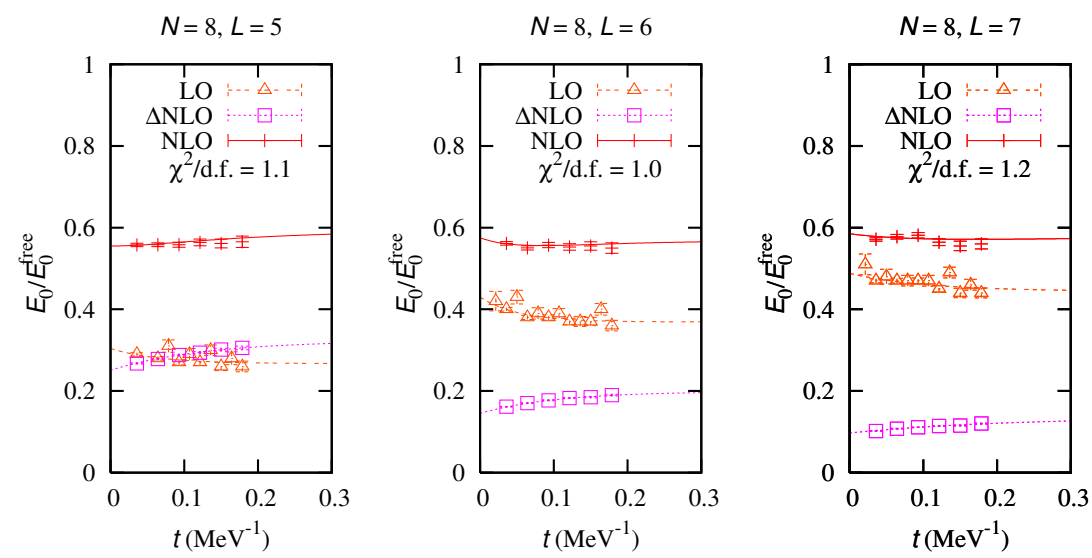

FIG. 2: Plots of the three energy ratios defined in Eq. (51) for $N=8$ and $L=5,6,7$. These are labelled as LO, $\Delta \mathrm{NLO}$, NLO respectively.
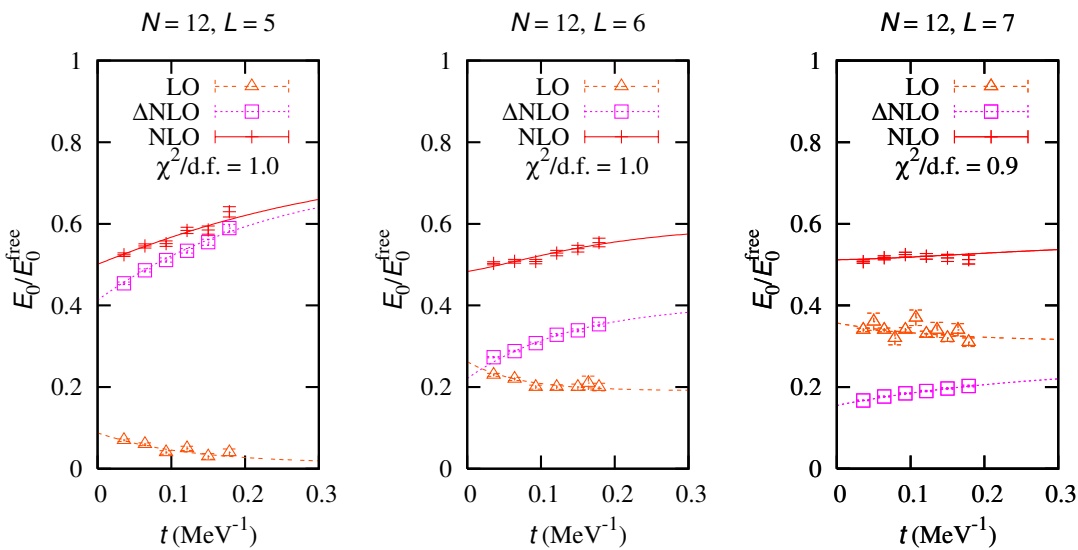

FIG. 3: Plots of the three energy ratios defined in Eq. (51) for $N=12$ and $L=5,6,7$. These are labelled as LO, $\triangle \mathrm{NLO}$, NLO respectively.

ground state and the lowest excited state. The reduced chi-square for each fit is shown in Fig. 2] and 3, and in each case they are close to 1.

We calculate the Fermi momentum $k_{F}$ for each neutron spin from the corresponding 


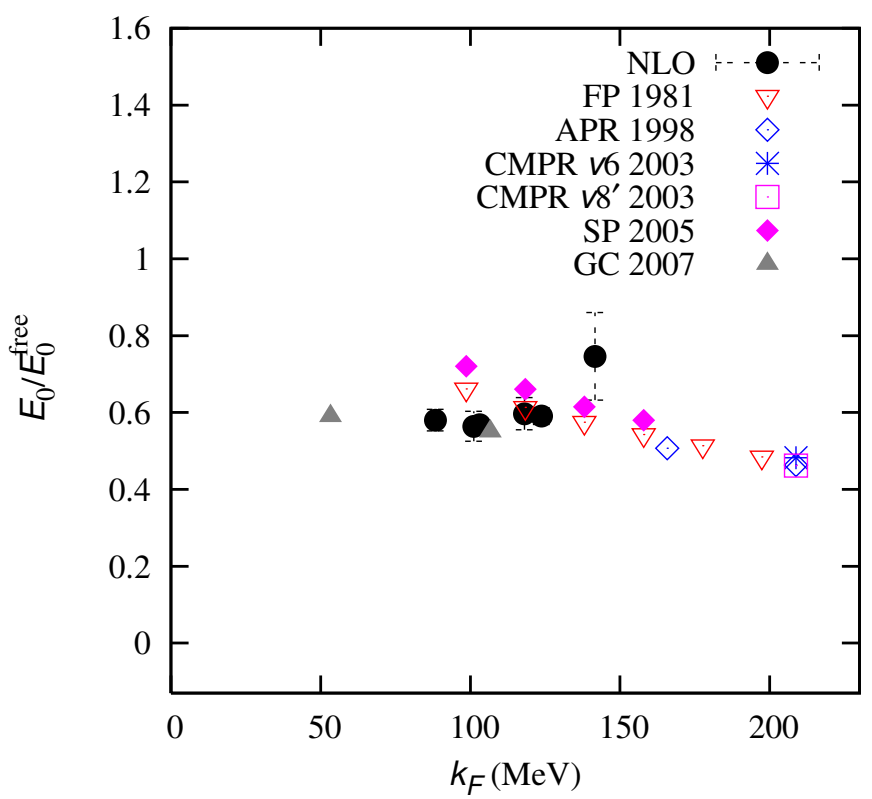

FIG. 4: Results for $E_{0, \mathrm{NLO}} / E_{0}^{\text {free }}$ versus Fermi momentum $k_{F}$. For comparison we show the results for FP 1981 [12], APR 1998 [13], CMPR $v 6$ and $v 8^{\prime} 2003$ [14], SP 2005 [15], and GC 2007 [16].

density. In our case $\rho_{\uparrow}=\rho_{\downarrow}=N /\left(2 L^{3}\right)$ and so

$$
k_{F}=\frac{1}{L}\left(3 \pi^{2} N\right)^{1 / 3}
$$

In Fig. 4 we show the results for $E_{0, \mathrm{NLO}} / E_{0}^{\text {free }}$ versus Fermi momentum $k_{F}$. The error bars on $E_{0, \mathrm{NLO}} / E_{0}^{\text {free }}$ represent uncertainties from the asymptotic fits in Eq. (52)-(154). For comparison we show other results from the literature: FP 1981 [12], APR 1998 [13], CMPR $v 6$ and $v 8^{\prime}$ [14], SP 2005 [15], and GC 2007 [16]. We find good agreement near $k_{F}=120$ $\mathrm{MeV}$ but there is disagreement whether the slope is positive or negative.

\section{ANALYSIS AND DISCUSSION}

Neutron matter at $k_{F} \sim 80 \mathrm{MeV}$ is close to the idealized unitarity limit, where the $S$-wave scattering length is infinite and the range of the interaction is negligible. At lower densities corrections due to the scattering length become more important, and at higher densities corrections due to the effective range and other effects become important. In the unitarity limit the ground state has no dimensionful parameters other than particle density and so the ground state energy of the system should obey a simple relation $E_{0}=\xi E_{0}^{\text {free }}$ for some 
dimensionless constant $\xi$. The universal nature of the unitarity limit endows it relevance to several areas of physics, and in atomic physics the unitarity limit has been studied extensively with ultracold ${ }^{6} \mathrm{Li}$ and ${ }^{40} \mathrm{~K}$ atoms using a magnetic-field Feshbach resonance [17, 18, 19, 20].

Recent experiments for $\xi$ have measured the expansion of ${ }^{6} \mathrm{Li}$ and ${ }^{40} \mathrm{~K}$ in the unitarity limit released from a harmonic trap. The measured values for $\xi$ are $0.51(4)$ [21], $0.46_{-05}^{+12}$ [22], and $0.32_{-13}^{+10}[23]$. The discrepancy between these measurements and larger values for $\xi$ reported in earlier experiments [24, 25, 26] suggests that further work may be needed.

There have been numerous analytic calculations of $\xi[27,28,29,30,31,32,33,34$, 35, 36]. The values for $\xi$ vary roughly from 0.2 to 0.6. Fixed-node Green's function Monte Carlo calculations have found $\xi$ to be $0.44(1)$ [37] and 0.42(1) [38]. An estimate based on KohnSham theory for the two-fermion system in a harmonic trap yields a value of 0.42 [39]. There have also been simulations of two-component fermions on the lattice in the unitarity limit at non-zero temperature. When data are extrapolated to zero temperature the results of [40] produce a value for $\xi$ similar to the fixed-node results. The same is true for [41, 42], though with significant error bars, while calculations by Lee and Schäfer [43, 44] established a bound, $0.07 \leq \xi \leq 0.42$.

For finite $S$-wave scattering length $a_{0}$ the deviation away from unitarity can be parameterized as

$$
\frac{E_{0}}{E_{0}^{\text {free }}} \approx \xi-\frac{\xi_{1}}{k_{F} a_{0}} .
$$

Both $\xi$ and $\xi_{1}$ have been computed using the lattice transfer matrix projection method discussed above. The results are $\xi=0.25(3)$ [8] and $\xi_{1}=1.0(1)$ [6]. More recent lattice calculations find similar values for $\xi$ and $\xi_{1}[45,46,47]$. There is general agreement in the recent literature on the value of $\xi_{1}$ [38, 48, 49]. Further work will be needed to resolve the remaining differences between the various calculations for $\xi$. We use the values from [8] and [6] in our analysis.

In addition to the corrections at finite scattering length we expect corrections proportional to $k_{F} r_{0}$ due to the $S$-wave effective range $r_{0}$. We also expect higher-order corrections away from the unitarity limit arising from higher powers of $1 /\left(k_{F} a_{0}\right)$ and $k_{F} r_{0}$, as well as other terms associated with the $S$-wave shape parameter and triplet $P$-wave scattering volumes. In Fig. 5 we show both $E_{0, \mathrm{LO}} / E_{0}^{\text {free }}$ and $E_{0, \mathrm{NLO}} / E_{0}^{\text {free }}$ versus $k_{F}$. For comparison we plot

$$
f\left(k_{F} a_{0}\right)=\xi-\frac{\xi_{1}}{k_{F} a_{0}}
$$




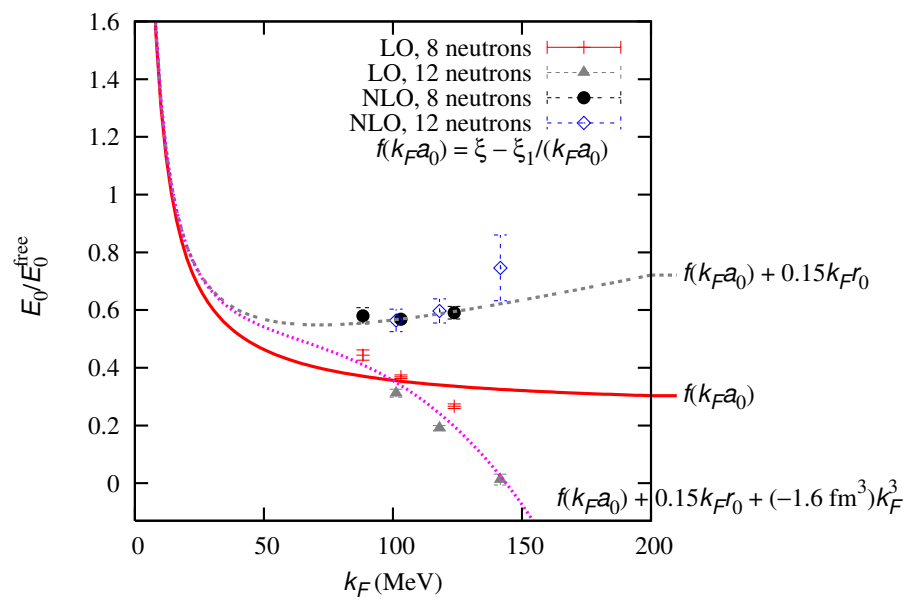

FIG. 5: Plot of $E_{0, \mathrm{LO}} / E_{0}^{\text {free }}$ and $E_{0, \mathrm{NLO}} / E_{0}^{\text {free }}$ versus $k_{F}$. For comparison we plot $f\left(k_{F} a_{0}\right)$, $f\left(k_{F} a_{0}\right)+0.15 k_{F} r_{0}$, and $f\left(k_{F} a_{0}\right)+0.15 k_{F} r_{0}+\left(-1.6 \mathrm{fm}^{3}\right) k_{F}^{3}$.

with $\xi=0.25, \xi_{1}=1.0$, and neutron scattering length $a_{0}=-18.5 \mathrm{fm}$. From Fig. 5 we see that the NLO energy ratio $E_{0, \mathrm{NLO}} / E_{0}^{\text {free }}$ is approximately described by

$$
E_{0, \mathrm{NLO}} / E_{0}^{\text {free }} \approx f\left(k_{F} a_{0}\right)+0.15 k_{F} r_{0}
$$

where $r_{0}$ is the neutron effective range $2.7 \mathrm{fm}$. The $k_{F} r_{0}$ term in Eq. (58) can be interpreted as the correction due to the neutron effective range. But as noted above there should also be corrections from higher powers of $1 /\left(k_{F} a_{0}\right)$ and $k_{F} r_{0}$ and from the $S$-wave shape parameter and triplet $P$-wave scattering volumes. It is not obvious why these higher-order effects are all numerically small at $k_{F} \approx m_{\pi}$ as the NLO lattice results suggest.

In contrast we see deviations beyond the $1 /\left(k_{F} a_{0}\right)$ and $k_{F} r_{0}$ corrections in the LO lattice results. As shown in Fig. 5 the leading-order ratio $E_{0, \mathrm{LO}} / E_{0}^{\text {free }}$ appears to lie on the curve

$$
E_{0, \mathrm{LO}} / E_{0}^{\text {free }} \approx f\left(k_{F} a_{0}\right)+0.15 k_{F} r_{0}+\left(-1.6 \mathrm{fm}^{3}\right) k_{F}^{3}
$$

We know from the ${ }^{1} S_{0}$ phase shifts in Fig. 1 that $S$-wave scattering for the LO and NLO actions are nearly identical. This explains the common coefficient of 0.15 in front of $k_{F} r_{0}$ for both LO and NLO results. Therefore, the difference between LO and NLO results must come from interactions in higher partial waves.

For the LO action each of triplet $P$-wave interactions in Fig. 1 are attractive. The $\left(-1.6 \mathrm{fm}^{3}\right) k_{F}^{3}$ term in Eq. (59) for the LO action is consistent with the type of correction 


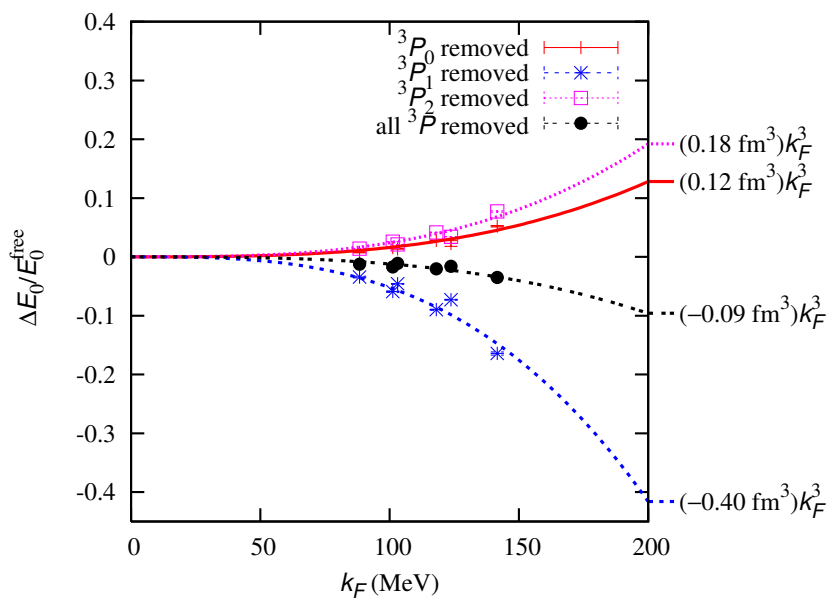

FIG. 6: The change $\Delta E_{0, \mathrm{NLO}} / E_{0}^{\text {free }}$ due to removing ${ }^{3} P_{0},{ }^{3} P_{1},{ }^{3} P_{2}$, or all triplet $P$-wave interactions.

we expect from the negative triplet $P$-wave scattering volumes. On the other hand, the $k_{F}^{3}$ correction from $P$-wave interactions in the NLO action seems to be numerically very small. To understand this better we probe the relation between low-energy $P$-wave interactions and the energy ratio $E_{0, \mathrm{NLO}} / E_{0}^{\text {free }}$ by varying coefficients of the NLO operators.

The NLO coefficients in Table \ were determined by fitting the five data points labelled by arrows in Fig. 1. We consider four variations of these NLO coefficients. For the first variation we set the phase shift for the ${ }^{3} P_{0}$ data point to zero while keeping other data points the same. For the second variation we zero out the phase shift of the ${ }^{3} P_{1}$ data point while keeping others the same. For the third variation we zero out only the ${ }^{3} P_{2}$ phase shift, and for the fourth we zero out all three triplet $P$-waves. The change $\Delta E_{0, \mathrm{NLO}} / E_{0}^{\text {free }}$ due to each of these variations is plotted in Fig. 6. The results show significant cancellation between the $P$-wave contributions. In fact the total contribution from all $P$-waves is smaller than any individual contribution. In Fig. 7 we show $E_{0, \mathrm{NLO}} / E_{0}^{\text {free }}$ and the effect of removing all triplet $P$-wave contributions. Both data sets lie approximately on the curve

$$
E_{0, \mathrm{NLO}} / E_{0}^{\mathrm{free}} \approx f\left(k_{F} a_{0}\right)+0.15 k_{F} r_{0}
$$

We see that the total effect of the triplet $P$-wave scattering volumes is small due to cancellations between the $J=0,1,2$ contributions. 


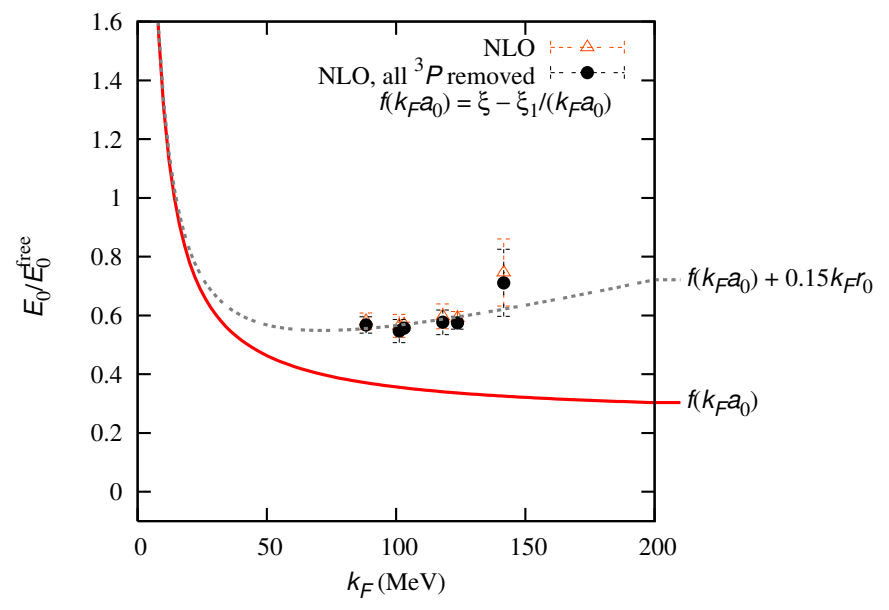

FIG. 7: Plot of $E_{0, \mathrm{NLO}} / E_{0}^{\text {free }}$ versus $k_{F}$ and the effect of removing all triplet $P$-wave contributions.

\section{SUMMARY}

We have discussed lattice simulations of the ground state of dilute neutron matter using chiral effective field theory at next-to-leading order. In the first paper the coefficients of the next-to-leading-order lattice action were determined by matching nucleon-nucleon scattering data for momenta up to the pion mass. In this second paper we used the same lattice action to simulate the ground state of up to 12 neutrons in a periodic cube using Monte Carlo for the density range from $2 \%$ to $8 \%$ of normal nuclear density. We found agreement near $k_{F}=120 \mathrm{MeV}$ for the ground energy ratio $E_{0} / E_{0}^{\text {free }}$ with results in the literature. However there is disagreement on whether the ratio is slightly increasing or slightly decreasing with $k_{F}$.

We analyzed the energy ratio as an expansion about the unitarity limit with corrections due to finite scattering length, effective range, and $P$-wave interactions. We find significant cancellation between the various triplet $P$-wave contributions to the ground state energy. We find a good fit to the lattice data using

$$
E_{0, \mathrm{NLO}} / E_{0}^{\mathrm{free}} \approx \xi-\frac{\xi_{1}}{k_{F} a_{0}}+0.15 k_{F} r_{0}
$$

with $\xi=0.25, \xi_{1}=1.0$. The coefficient in front of $k_{F} r_{0}$ should be a universal constant and therefore measurable in other quantum systems near the unitarity limit. In future studies we will consider larger systems of dilute neutron matter and test model independence of results in the manner discussed in [1] using several different lattice actions. 


\section{Acknowledgements}

Partial financial support from the Deutsche Forschungsgemeinschaft (SFB/TR 16), Helmholtz Association (contract number VH-NG-222 and VH-VI-231), and U.S. Department of Energy (DE-FG02-03ER41260) are gratefully acknowledged. This research is part of the EU Integrated Infrastructure Initiative in Hadron Physics under contract number RII3-CT-2004-506078. The computational resources for this project were provided by the John von Neumann Institute for Computing at the Forschungszentrum Jülich.

\section{APPENDIX A: LATTICE ACTION}

\section{Notation}

We assume exact isospin symmetry and neglect electromagnetic interactions. $\vec{n}$ represents integer-valued lattice vectors on a three-dimensional spatial lattice, and $\vec{p}, \vec{q}, \vec{k}$ represent integer-valued momentum lattice vectors. $\hat{l}=\hat{1}, \hat{2}, \hat{3}$ are unit lattice vectors in the spatial directions, $a$ is the spatial lattice spacing, and $L$ is the length of the cubic spatial lattice in each direction. The lattice time step is $a_{t}$, and $n_{t}$ labels the number of time steps. We define $\alpha_{t}$ as the ratio between lattice spacings, $\alpha_{t}=a_{t} / a$. Throughout we use dimensionless parameters and operators, which correspond with physical values multiplied by the appropriate power of $a$. Final results are presented in physical units with the corresponding unit stated explicitly.

We use $a$ and $a^{\dagger}$ to denote annihilation and creation operators. We make explicit all spin and isospin indices,

$$
\begin{aligned}
& a_{0,0}=a_{\uparrow, p}, \quad a_{0,1}=a_{\uparrow, n}, \\
& a_{1,0}=a_{\downarrow, p}, \quad a_{1,1}=a_{\downarrow, n} .
\end{aligned}
$$

The first subscript is for spin and the second subscript is for isospin. We use $\tau_{I}$ with $I=1,2,3$ to represent Pauli matrices acting in isospin space and $\sigma_{S}$ with $S=1,2,3$ to represent Pauli matrices acting in spin space.

We use the eight vertices of a unit cube on the lattice to define spatial derivatives. For 
each spatial direction $l=1,2,3$ and any lattice function $f(\vec{n})$, let

$$
\Delta_{l} f(\vec{n})=\frac{1}{4} \sum_{\nu_{1}, \nu_{2}, \nu_{3}=0,1}(-1)^{\nu_{l}+1} f(\vec{n}+\vec{\nu}), \quad \vec{\nu}=\nu_{1} \hat{1}+\nu_{2} \hat{2}+\nu_{3} \hat{3} .
$$

We also define the double spatial derivative along direction $l$,

$$
\nabla_{l}^{2} f(\vec{n})=f(\vec{n}+\hat{l})+f(\vec{n}-\hat{l})-2 f(\vec{n})
$$

\section{Local densities and currents}

We define the local density,

$$
\rho^{a^{\dagger}, a}(\vec{n})=\sum_{i, j=0,1} a_{i, j}^{\dagger}(\vec{n}) a_{i, j}(\vec{n})
$$

which is invariant under Wigner's SU(4) symmetry [50]. Similarly we define the local spin density for $S=1,2,3$,

$$
\rho_{S}^{a^{\dagger}, a}(\vec{n})=\sum_{i, j, i^{\prime}=0,1} a_{i, j}^{\dagger}(\vec{n})\left[\sigma_{S}\right]_{i i^{\prime}} a_{i^{\prime}, j}(\vec{n}),
$$

isospin density for $I=1,2,3$,

$$
\rho_{I}^{a^{\dagger}, a}(\vec{n})=\sum_{i, j, j^{\prime}=0,1} a_{i, j}^{\dagger}(\vec{n})\left[\tau_{I}\right]_{j j^{\prime}} a_{i, j^{\prime}}(\vec{n}),
$$

and spin-isospin density for $S, I=1,2,3$,

$$
\rho_{S, I}^{a^{\dagger}, a}(\vec{n})=\sum_{i, j, i^{\prime}, j^{\prime}=0,1} a_{i, j}^{\dagger}(\vec{n})\left[\sigma_{S}\right]_{i i^{\prime}}\left[\tau_{I}\right]_{j j^{\prime}} a_{i^{\prime}, j^{\prime}}(\vec{n}) .
$$

For each static density we also have an associated current density. Similar to the definition of the lattice derivative $\Delta_{l}$ in (A3), we use the eight vertices of a unit cube,

$$
\vec{\nu}=\nu_{1} \hat{1}+\nu_{2} \hat{2}+\nu_{3} \hat{3}
$$

for $\nu_{1}, \nu_{2}, \nu_{3}=0,1$. Let $\vec{\nu}(-l)$ for $l=1,2,3$ be the result of reflecting the $l^{\text {th }}$-component of $\vec{\nu}$ about the center of the cube,

$$
\vec{\nu}(-l)=\vec{\nu}+\left(1-2 \nu_{l}\right) \hat{l}
$$

Omitting factors of $i$ and $1 / m$, we can write the $l^{\text {th }}$-component of the SU(4)-invariant current density as

$$
\Pi_{l}^{a^{\dagger}, a}(\vec{n})=\frac{1}{4} \sum_{\nu_{1}, \nu_{2}, \nu_{3}=0,1} \sum_{i, j=0,1}(-1)^{\nu_{l}+1} a_{i, j}^{\dagger}(\vec{n}+\vec{\nu}(-l)) a_{i, j}(\vec{n}+\vec{\nu})
$$


Similarly the $l^{\text {th }}$-component of spin current density is

$$
\Pi_{l, S}^{a^{\dagger}, a}(\vec{n})=\frac{1}{4} \sum_{\nu_{1}, \nu_{2}, \nu_{3}=0,1} \sum_{i, j, i^{\prime}=0,1}(-1)^{\nu_{l}+1} a_{i, j}^{\dagger}(\vec{n}+\vec{\nu}(-l))\left[\sigma_{S}\right]_{i i^{\prime}} a_{i^{\prime}, j}(\vec{n}+\vec{\nu}),
$$

$l^{\text {th }}$-component of isospin current density is

$$
\Pi_{l, I}^{a^{\dagger}, a}(\vec{n})=\frac{1}{4} \sum_{\nu_{1}, \nu_{2}, \nu_{3}=0,1} \sum_{i, j, j^{\prime}=0,1}(-1)^{\nu_{l}+1} a_{i, j}^{\dagger}(\vec{n}+\vec{\nu}(-l))\left[\tau_{I}\right]_{j j^{\prime}} a_{i, j^{\prime}}(\vec{n}+\vec{\nu}),
$$

and $l^{\text {th }}$-component of spin-isospin current density is

$$
\Pi_{l, S, I}^{a^{\dagger}, a}(\vec{n})=\frac{1}{4} \sum_{\nu_{1}, \nu_{2}, \nu_{3}=0,1} \sum_{i, j, i^{\prime}, j^{\prime}=0,1}(-1)^{\nu_{l}+1} a_{i, j}^{\dagger}(\vec{n}+\vec{\nu}(-l))\left[\sigma_{S}\right]_{i i^{\prime}}\left[\tau_{I}\right]_{j j^{\prime}} a_{i^{\prime}, j^{\prime}}(\vec{n}+\vec{\nu})
$$

\section{Instantaneous free pion action}

The lattice action for free pions with purely instantaneous propagation is

$$
S_{\pi \pi}\left(\pi_{I}\right)=\alpha_{t}\left(\frac{m_{\pi}^{2}}{2}+3\right) \sum_{\vec{n}, n_{t}, I} \pi_{I}\left(\vec{n}, n_{t}\right) \pi_{I}\left(\vec{n}, n_{t}\right)-\alpha_{t} \sum_{\vec{n}, n_{t}, I, l} \pi_{I}\left(\vec{n}, n_{t}\right) \pi_{I}\left(\vec{n}+\hat{l}, n_{t}\right)
$$

where $\pi_{I}$ is the pion field labelled with isospin index $I$. It is convenient to define a rescaled pion field, $\pi_{I}^{\prime}$,

$$
\begin{gathered}
\pi_{I}^{\prime}\left(\vec{n}, n_{t}\right)=\sqrt{q_{\pi}} \pi_{I}\left(\vec{n}, n_{t}\right), \\
q_{\pi}=\alpha_{t}\left(m_{\pi}^{2}+6\right) .
\end{gathered}
$$

Then

$$
S_{\pi \pi}\left(\pi_{I}^{\prime}\right)=\frac{1}{2} \sum_{\vec{n}, n_{t}, I} \pi_{I}^{\prime}\left(\vec{n}, n_{t}\right) \pi_{I}^{\prime}\left(\vec{n}, n_{t}\right)-\frac{\alpha_{t}}{q_{\pi}} \sum_{\vec{n}, n_{t}, I, l} \pi_{I}^{\prime}\left(\vec{n}, n_{t}\right) \pi_{I}^{\prime}\left(\vec{n}+\hat{l}, n_{t}\right) .
$$

In momentum space the action is

$$
S_{\pi \pi}\left(\pi_{I}^{\prime}\right)=\frac{1}{L^{3}} \sum_{I, \vec{k}} \pi_{I}^{\prime}\left(-\vec{k}, n_{t}\right) \pi_{I}^{\prime}\left(\vec{k}, n_{t}\right)\left[\frac{1}{2}-\frac{\alpha_{t}}{q_{\pi}} \sum_{l} \cos \left(\frac{2 \pi k_{l}}{L}\right)\right] .
$$

The instantaneous pion correlation function at spatial separation $\vec{n}$ is

$$
\begin{aligned}
\left\langle\pi_{I}^{\prime}\left(\vec{n}, n_{t}\right) \pi_{I}^{\prime}\left(\overrightarrow{0}, n_{t}\right)\right\rangle & \left.=\frac{\int D \pi_{I}^{\prime} \pi_{I}^{\prime}\left(\vec{n}, n_{t}\right) \pi_{I}^{\prime}\left(\overrightarrow{0}, n_{t}\right) \exp \left[-S_{\pi \pi}\right]}{\int D \pi_{I}^{\prime} \exp \left[-S_{\pi \pi}\right]} \text { (no sum on } I\right) \\
& =\frac{1}{L^{3}} \sum_{\vec{k}} e^{-i \frac{2 \pi}{L} \vec{k} \cdot \vec{n}} D_{\pi}(\vec{k}),
\end{aligned}
$$


where

$$
D_{\pi}(\vec{k})=\frac{1}{1-\frac{2 \alpha_{t}}{q_{\pi}} \sum_{l} \cos \left(\frac{2 \pi k_{l}}{L}\right)} .
$$

It is useful also to define the two-derivative pion correlator, $G_{S_{1} S_{2}}(\vec{n})$,

$$
\begin{aligned}
G_{S_{1} S_{2}}(\vec{n}) & \left.=\left\langle\Delta_{S_{1}} \pi_{I}^{\prime}\left(\vec{n}, n_{t}\right) \Delta_{S_{2}} \pi_{I}^{\prime}\left(\overrightarrow{0}, n_{t}\right)\right\rangle \quad \text { (no sum on } I\right) \\
& =\frac{1}{16} \sum_{\nu_{1}, \nu_{2}, \nu_{3}=0,1} \sum_{\nu_{1}^{\prime}, \nu_{2}^{\prime}, \nu_{3}^{\prime}=0,1}(-1)^{\nu_{S_{1}}}(-1)^{\nu_{S_{2}}^{\prime}}\left\langle\pi_{I}^{\prime}\left(\vec{n}+\vec{\nu}-\vec{\nu}^{\prime}, n_{t}\right) \pi_{I}^{\prime}\left(\overrightarrow{0}, n_{t}\right)\right\rangle .
\end{aligned}
$$

\section{Leading-order transfer matrix $\mathbf{L O}_{2}$}

We use the $O\left(a^{4}\right)$-improved free lattice Hamiltonian,

$$
\begin{aligned}
H_{\text {free }} & =\frac{49}{12 m} \sum_{\vec{n}} \sum_{i, j=0,1} a_{i, j}^{\dagger}(\vec{n}) a_{i, j}(\vec{n}) \\
& -\frac{3}{4 m} \sum_{\vec{n}} \sum_{i, j=0,1} \sum_{l=1,2,3}\left[a_{i, j}^{\dagger}(\vec{n}) a_{i, j}(\vec{n}+\hat{l})+a_{i, j}^{\dagger}(\vec{n}) a_{i, j}(\vec{n}-\hat{l})\right] \\
& +\frac{3}{40 m} \sum_{\vec{n}} \sum_{i, j=0,1} \sum_{l=1,2,3}\left[a_{i, j}^{\dagger}(\vec{n}) a_{i, j}(\vec{n}+2 \hat{l})+a_{i, j}^{\dagger}(\vec{n}) a_{i, j}(\vec{n}-2 \hat{l})\right] \\
& -\frac{1}{180 m} \sum_{\vec{n}} \sum_{i, j=0,1} \sum_{l=1,2,3}\left[a_{i, j}^{\dagger}(\vec{n}) a_{i, j}(\vec{n}+3 \hat{l})+a_{i, j}^{\dagger}(\vec{n}) a_{i, j}(\vec{n}-3 \hat{l})\right] .
\end{aligned}
$$

The leading-order transfer matrix designated $M_{\mathrm{LO}_{2}}$ in [7] is

$$
\begin{aligned}
M_{\mathrm{LO}_{2}} & =: \exp \left\{-H_{\mathrm{free}} \alpha_{t}-\frac{\alpha_{t}}{2 L^{3}} \sum_{\vec{q}} f\left(q^{2}\right)\left[C \rho^{a^{\dagger}, a}(\vec{q}) \rho^{a^{\dagger}, a}(-\vec{q})+C_{I^{2}} \sum_{I} \rho_{I}^{a^{\dagger}, a}(\vec{q}) \rho_{I}^{a^{\dagger}, a}(-\vec{q})\right]\right. \\
& \left.+\frac{g_{A}^{2} \alpha_{t}^{2}}{8 f_{\pi}^{2} q_{\pi}} \sum_{S_{1}, S_{2}, I} \sum_{\vec{n}_{1}, \vec{n}_{2}} G_{S_{1} S_{2}}\left(\vec{n}_{1}-\vec{n}_{2}\right) \rho_{S_{1}, I}^{a^{\dagger}, a}\left(\vec{n}_{1}\right) \rho_{S_{2}, I}^{a^{\dagger}, a}\left(\vec{n}_{2}\right)\right\}
\end{aligned}
$$

where the momentum-dependent coefficient function $f\left(q^{2}\right)$ is defined as

$$
f\left(q^{2}\right)=f_{0}^{-1} \exp \left[-b \sum_{l}\left(1-\cos q_{l}\right)\right],
$$

and the normalization factor $f_{0}$ is determined by the condition

$$
f_{0}=\frac{1}{L^{3}} \sum_{\vec{q}} \exp \left[-b \sum_{l}\left(1-\cos q_{l}\right)\right] .
$$


The value $b=0.6$ gives approximately the correct average effective range for the two $S$-wave channels when $C$ and $C_{I^{2}}$ are properly tuned. $C$ is the coefficient of the Wigner SU(4)invariant contact interaction and $C_{I^{2}}$ is the coefficient of the isospin-dependent contact interaction. For $C$ and $C_{I^{2}}$ we use the values

$$
\begin{aligned}
& C=\left(3 C^{I=1}+C^{I=0}\right) / 4, \\
& C_{I^{2}}=\left(C^{I=1}-C^{I=0}\right) / 4,
\end{aligned}
$$

with $C^{I=1}=-3.414 \times 10^{-5} \mathrm{MeV}^{-2}$ and $C^{I=0}=-4.780 \times 10^{-5} \mathrm{MeV}^{-2}$.

[1] B. Borasoy, E. Epelbaum, H. Krebs, D. Lee, and U.-G. Meißner (2007).

[2] C. J. Pethick and D. G. Ravenhall, Ann. Rev. Nucl. Part. Sci. 45, 429 (1995).

[3] J. M. Lattimer and M. Prakash, Science 304, 536 (2004), astro-ph/0405262.

[4] B. Borasoy, E. Epelbaum, H. Krebs, D. Lee, and U.-G. Meißner, Eur. Phys. J. A34, 185 (2007), arXiv:0708.1780 [nucl-th].

[5] V. G. J. Stoks, R. A. M. Kompl, M. C. M. Rentmeester, and J. J. de Swart, Phys. Rev. C48, $792(1993)$.

[6] D. Lee, Phys. Rev. B75, 134502 (2007), cond-mat/0606706.

[7] B. Borasoy, E. Epelbaum, H. Krebs, D. Lee, and U.-G. Meißner, Eur. Phys. J. A31, 105 (2007), nucl-th/0611087.

[8] D. Lee, Phys. Rev. B73, 115112 (2006), cond-mat/0511332.

[9] D. Lee, Phys. Rev. C71, 044001 (2005), nucl-th/0407101.

[10] J.-W. Chen, D. Lee, and T. Schäfer, Phys. Rev. Lett. 93, 242302 (2004), nucl-th/0408043.

[11] D. Lee, Phys. Rev. Lett. 98, 182501 (2007), nucl-th/0701041.

[12] B. Friedman and V. R. Pandharipande, Nucl. Phys. A361, 502 (1981).

[13] A. Akmal, V. R. Pandharipande, and D. G. Ravenhall, Phys. Rev. C58, 1804 (1998), nuclth/9804027.

[14] J. Carlson, J. Morales, J., V. R. Pandharipande, and D. G. Ravenhall, Phys. Rev. C68, 025802 (2003), nucl-th/0302041.

[15] A. Schwenk and C. J. Pethick, Phys. Rev. Lett. 95, 160401 (2005), nucl-th/0506042.

[16] A. Gezerlis and J. Carlson (2007), arXiv:0711.3006 [nucl-th]. 
[17] E. Tiesinga, B. J. Verhaar, and H. T. C. Stoof, Phys. Rev. A47, 4114 (1993).

[18] W. C. Stwalley, Phys. Rev. Lett. 37, 1628 (1976).

[19] P. Courteille, R. S. Freeland, D. J. Heinzen, F. A. van Abeelen, and B. J. Verhaar, Phys. Rev. Lett. 81, 69 (1998).

[20] S. Inouye, M. R. Andrews, J. Stenger, H.-J. Miesner, D. Stamper-Kurn, and W. Ketterle, Nature 392, 151 (1998).

[21] J. Kinast, A. Turlapov, J. E. Thomas, Q. Chen, J. Stajic, and K. Levin, Science 307, 1296 (2005), cond-mat/0502087.

[22] J. T. Stewart, J. P. Gaebler, C. A. Regal, and D. S. Jin, Phys. Rev. Lett. 97, 220406 (2006), arXiv.org:cond-mat/0607776.

[23] M. Bartenstein, A. Altmeyer, S. Riedl, S. Jochim, C. Chin, J. Hecker Denschlag, and R. Grimm, Phys. Rev. Lett. 92, 120401 (2004).

[24] K. M. O’Hara, S. L. Hemmer, M. E. Gehm, S. R. Granade, and J. E. Thomas, Science 298, 2179 (2002).

[25] T. Bourdel, J. Cubizolles, L. Khaykovich, K. M. F. Magalhaes, S. J. J. M. F. Kokkelmans, G. V. Shlyapnikov, and C. Salomon, Phys. Rev. Lett. 91, 020402 (2003).

[26] M. E. Gehm, S. L. Hemmer, S. R. Granade, K. M. O'Hara, and J. E. Thomas, Phys. Rev. A68, 011401(R) (2003).

[27] J. R. Engelbrecht, M. Randeria, and C. S. de Melo, Phys. Rev. B55, 15153 (1997).

[28] G. A. Baker, Phys. Rev. C60, 054311 (1999).

[29] H. Heiselberg, Phys. Rev. A 63, 043606 (2001), cond-mat/0002056.

[30] A. Perali, P. Pieri, and G. C. Strinati, Phys. Rev. Lett. 93, 100404 (2004).

[31] T. Schäfer, C.-W. Kao, and S. R. Cotanch, Nucl. Phys. A762, 82 (2005), nucl-th/0504088.

[32] Y. Nishida and D. T. Son, Phys. Rev. Lett. 97, 050403 (2006), cond-mat/0604500.

[33] Y. Nishida and D. T. Son, Phys. Rev. A 75, 063617 (2007), cond-mat/0607835.

[34] P. Arnold, J. E. Drut, and D. T. Son, Phys. Rev. A 75, 043605 (2007), cond-mat/0608477.

[35] P. Nikolic and S. Sachdev, Phys. Rev. A 75, 033608 (2007), cond-mat/0609106.

[36] M. Y. Veillette, D. E. Sheehy, and L. Radzihovsky, Phys. Rev. A 75, 043614 (2007), condmat/0610798.

[37] J. Carlson, S. Y. Chang, V. R. Pandharipande, and K. Schmidt, Phys. Rev. Lett. 91, 50401 (2003), physics/0303094. 
[38] G. E. Astrakharchik, J. Boronat, J. Casulleras, and S. Giorgini, Phys. Rev. Lett. 93, 200404 (2004), cond-mat/0406113.

[39] T. Papenbrock, Phys. Rev. A 72, 041603 (2005), cond-mat/0507183.

[40] A. Bulgac, J. E. Drut, and P. Magierski, Phys. Rev. Lett. 96, 090404 (2006), condmat/0505374.

[41] E. Burovski, N. Prokofev, B. Svistunov, and M. Troyer, Phys. Rev. Lett. 96, 160402 (2006), cond-mat/0602224.

[42] E. Burovski, N. Prokofev, B. Svistunov, and M. Troyer, New J. Phys. 8, 153 (2006), condmat/0605350.

[43] D. Lee and T. Schäfer, Phys. Rev. C73, 015201 (2006), nucl-th/0509017.

[44] D. Lee and T. Schäfer, Phys. Rev. C73, 015202 (2006), nucl-th/0509018.

[45] D. Lee (2007), arXiv.org:0704.3439.

[46] T. Abe and R. Seki (2007), arXiv:0708.2523 [nucl-th].

[47] T. Abe and R. Seki (2007), arXiv:0708.2524 [nucl-th].

[48] S. Y. Chang, V. R. Pandharipande, J. Carlson, and K. E. Schmidt, Phys. Rev. A70, 043602 (2004).

[49] J.-W. Chen and E. Nakano, Phys. Rev. A75, 043620 (2007), cond-mat/0610011.

[50] E. Wigner, Phys. Rev. 51, 106 (1937). 